included a discussion on risk assessment and management, signposting to appropriate resources and sharing learning points. Confidence in history-taking, psychosocial assessment and management were assessed by self-report before and after the simulation.

Results Following both the self-harm and anorexia nervosa scenarios respectively, there were increases in clinician-reported confidence in history taking $(37 \%$ vs. $85.2 \% ; 41.7 \%$ vs. $70 \%)$, psychosocial assessment $(34.6 \%$ vs. $67.8 \%)$ and management (37\% vs. $60.7 \% ; 58.3 \%$ vs. $63.7 \%)$. Thematic analysis of qualitative feedback highlighted the desire for further training as well as various benefits of simulation.

Conclusion As the mental health needs of CYP continue to rise, it is clear that there remains an urgent and unmet need for mental health training for paediatric clinicians. This data suggests that low technology high fidelity simulation is a feasible method of improving clinician confidence in the assessment and management of CYP with mental health presentations. It is hoped that by developing such programmes, clinicians will be helped to cultivate the necessary skills to appropriately assess and manage patients presenting with mental health concerns.

\section{G261(P) HOW CONFIDENT DO PAEDIATRICIANS FEEL IN MANAGING MEDICALLY UNEXPLAINED SYMPTOMS?}

J Hoddes, I Takon. Paediatrics, East and North Hertfordshire NHS Trust, Stevenage, UK

\subsection{6/archdischild-2020-rcpch.225}

Aims This survey was designed to explore the experience and confidence of Paediatricians in the management of children and young people with Medically Unexplained Symptoms (MUS).

Methods Semi- structured questionnaires using mostly closed ended questions regarding clinicians' experience of managing cases of MUS and their confidence in managing such cases were distributed to all middle grade doctors and consultants working in acute and community paediatrics within our trust Results 17 completed questionnaires were analysed: 6 Middle Grade Doctors (MGD); 7 Hospital Consultants (HC) and 4 Community Consultants (CC). Data was subcategorised and frequencies of responses determined.

5/17 respondents, all hospital based, reported seeing children with MUS on a monthly basis.

$5 / 17$ respondents were confident in making a diagnosis of MUS (4 hospital and 1 community consultant). All Middle Grade Doctors did not feel confident in diagnosing MUS and all but one Middle Grade respondent felt uncomfortable discussing MUS with patients and families.

9/17 ( 53\%) of respondents strongly agreed with having an end point for investigations with 5 out of 9 respondents being hospital consultants.

Hospital Consultants and Middle Grade Doctors tended to believe that it was their role to rule out a physical cause before addressing mental health issues however Community Consultants were less likely to agree with this view.

The general trend amongst Paediatricians was towards sharing uncertainties about the diagnosis with the family but there was a diverse range of opinion on this irrespective of workplace or level of experience. All groups would raise the possibility of MUS with colleagues earlier than they would with the families.
Consultants (HC and CC) were generally likely to pre-empt normal test results with the family.

Community Consultants were the group most likely to feel that they had already received some training in MUS, however, several CC confused the diagnosis of MUS with Factitious Induced Illness in their comments.

Conclusions MUS causes high levels of morbidity within the paediatric population; however, our survey has shown that many Paediatricians of all levels do not feel confident in managing MUS. We advocate that training in Medically Unexplained Symptoms is mandatory for all Paediatricians.

\section{G262(P) MENTAL HEALTH MATTERS: THE IMPACT OF INCLUDING MENTAL HEALTH PROFESSIONALS WITHIN A GP-BASED CHILD HEALTH MULTIDISCIPLINARY TEAM}

I Talintyre, S Adnan, K Aitchison. Integrated Paediatrics, St Mary's Hospital, London, UK

\subsection{6/archdischild-2020-rcpch.226}

Aim The majority of mental health problems start in childhood and adolescence, but 3 in 4 children with a diagnosable mental health condition do not get access to the support they need. ${ }^{1}$ In order to address this deficit, we aimed to evaluate the impact of including mental health (CAMHS) professionals within a GP-based child health multidisciplinary team (MDT). A component of an existing child health GP hub model, these monthly MDT meetings are opportunities for GPs, specialists and allied healthcare professionals to discuss paediatric cases in primary care, providing integrated care with previously demonstrated increased connections and shared learning between professionals. $^{2}$

Methods 2 questionnaires were developed for GPs at 2 hub MDTs and distributed just prior to and 9 months after the introduction of a CAMHS professional. The attending CAMHS professional gave written feedback on their experience.

Results 14 questionnaires were returned by GPs. Since the inclusion of a CAMHS professional within the MDT, 100\% agreed/strongly agreed they added value to case discussions and felt comfortable contacting them for advice outside the MDT, and 75\% agreed their own awareness of services and resources available had increased.

Both pre- and post-intervention, 50\% of GPs disagreed/ strongly disagreed that they were confident managing paediatric patients with mental health problems on their own. The most commonly reported barriers to managing these patients in the community related to access (60\%) and waiting time (40\%) for secondary care.

The main themes in the CAMHS professional's reflections of attending the MDT were collaboration, building relationships with referrers, and mutual learning.

Conclusion The inclusion of a CAMHS professional in the child health hub MDT improved relationship-building and knowledge-sharing between professionals. However, GPs continue to lack confidence managing paediatric mental health. Further innovation is required to tackle barriers to accessing support including waiting time and limited resources.

\section{REFERENCES}

1. YoungMinds. YoungMinds Impact Report 2018-19. Available from: https://youngminds.org.uk/about-us/reports/our-impact-reports/

2. Montgomery-Taylor S, Watson M, Klaber R. Child Health General Practice Hubs: service evaluation. Archives of Disease in Childhood. 2016;101:333-337. 